\title{
Stimulus-Dependent Modulation of Suppressive Influences in MT
}

\author{
J. Nicholas Hunter ${ }^{1,2}$ and Richard T. Born ${ }^{1}$ \\ ${ }^{1}$ Department of Neurobiology, Harvard Medical School, Boston, Massachusetts 02115, and 2 Program in Neuroscience, Boston, Massachusetts 02115
}

Surround suppression contributes to important functions in visual processing, such as figure-ground segregation; however, this benefit comes at the cost of decreased neuronal sensitivity. Studies of receptive fields at several levels of the visual hierarchy have demonstrated that surround suppression is reduced for low contrast stimuli, thereby improving neuronal sensitivity. We investigated whether this reduction of surround suppression reflects a general processing strategy to boost sensitivity for weak signals by summing them over a larger region of the visual field (spatial integration) or if the reduction is limited to specialized stimulus conditions. To do this, we used stochastic motion stimuli to measure surround suppression in area MT of alert macaque monkeys. While varying stimulus size we also varied the strength of two other critical stimulus features: contrast and coherence (i.e., the proportion of dots moving in the preferred direction of the neuron). We found that reducing stimulus contrast weakened surround suppression, but reducing stimulus coherence had the opposite effect, indicating that diminished surround suppression is not a universal response to stimuli of low signal-to-noise. This can be partially explained by our other finding, which is that surrounds in MT are very broadly direction tuned. Instead of producing a reduction of surround suppression that would improve the ability of the neuron to integrate preferred direction motion, low coherence stimuli activated the broadly tuned surrounds relatively better than the centers, which are generally more direction selective. Our results are consistent with a normalization mechanism of surround suppression that pools broadly across multiple stimulus dimensions.

\section{Introduction}

Center-surround organization is a common feature of sensory receptive fields. The center region, or "classical receptive field," is encircled or flanked by regions that do not elicit spikes when stimulated alone but modulate neuronal activity when paired with a center stimulus. Surrounds are most commonly suppressive, which allows neurons to operate as differentiators, thereby removing redundancy from the inputs (Barlow, 1961) and selectively signaling discontinuity across the input space. Such a mechanism may be useful for figure-ground segregation (Allman et al., 1985b; Born et al., 2000) and the computation of three-dimensional shape from relative motion (Buracas and Albright, 1996).

Although computationally useful, suppressive surrounds are detrimental for processing noisy stimuli, because they limit the area over which stimulus features can be integrated to improve sensitivity. Horace Barlow (1959) first suggested that one way retinal ganglion cells might overcome this problem is by modulating their surrounds: strengthening surround suppression under photopic conditions, but weakening or eliminating it under

Received Aug. 31, 2010; revised 0ct. 5, 2010; accepted 0ct. 10, 2010.

This work was supported by National Eye Institute Grant EY11379 (R.T.B.), National Eye Institute Vision Core Grant EY12196, and a Kwan Fellowship (J.N.H.). This work comprised a portion of the PhD dissertation of J.N.H. We thank Phillip Hendrickson for excellent technical assistance and, for comments on this manuscript, John Assad, Marge Livingstone, Chris Pack, and James Tsui.

Correspondence should be addressed to Richard T. Born at the above address. E-mail: richard_born@ hms.harvard.edu.

DOI:10.1523/JNEUROSCI.4560-10.2011

Copyright $\odot 2011$ the authors $\quad 0270-6474 / 11 / 310678-09 \$ 15.00 / 0$ scotopic conditions (Barlow et al., 1957). Evidence that the visual cortex also employs such a strategy has come mainly from experiments in which stimulus contrast has been manipulated to alter signal strength. Neurons in V1 (Levitt and Lund, 1997; Polat et al., 1998; Kapadia et al., 1999; Sceniak et al., 1999; Cavanaugh et al., 2002) and MT (Pack et al., 2005) generally exhibit weaker surround suppression as stimulus contrast is decreased. In V1, oriented texture noise has also been shown to decrease surround suppression (Kapadia et al., 1999), thus suggesting that other conditions that weaken relevant signals might lead to adaptive changes in receptive field structure. In this case, however, it could be argued that adding the texture elements to the surround simply lowered the stimulus contrast.

We investigated whether the modulation of surround strength generalizes to other types of weak signals by using variable coherence random dot stimuli (Morgan and Ward, 1980), which allowed us to systematically vary the strength of the motion signal while keeping the overall contrast constant. Reducing the signal-to-noise ratio in this way leads to a decrease in psychophysical performance for both humans and monkeys (Morgan and Ward, 1980; Britten et al., 1992), but observers can improve their performance by integrating over both space and time (van Doorn and Koenderink, 1984; Watamaniuk and Sekuler, 1992). We thus hypothesized that MT neurons would show reduced surround suppression for low coherence motion stimuli.

Contrary to our hypothesis, we found that low coherence stimuli tended to increase the relative strength of suppressive surrounds, indicating that noisy stimuli do not always produce 
greater integration at the single-neuron level. Additional experiments revealed that this is most likely the result of much poorer direction selectivity in the surround, which allows the nonpreferred directions of motion present in low coherence stimuli to more effectively activate the suppressive surround.

\section{Materials and Methods}

\section{Electrophysiology}

Tungsten microelectrodes (Microprobe or FHC) were used to record extracellularly from random dot patterns to match the preferred features of the cell. Data were collected from three adult, male rhesus monkeys (Macaca mulatta). For the size tuning experiments, 18 cells were collected from monkey C, 4 cells from monkey L, and 31 cells from monkey $\mathrm{O}$. For the surround direction tuning experiments, 24 cells were collected from monkey C, 14 cells from monkey L, and 44 from monkey O. No significant differences were observed between monkeys, so data were pooled across animals.

\section{Visual stimuli}

Visual stimuli were presented on a monitor subtending $50 \times 37^{\circ}$ at a viewing distance of $39 \mathrm{~cm}$. Stimuli were drawn with MATLAB (MathWorks) using the Cogent Graphics toolbox developed by John Romaya (Laboratory of Neurobiology at the Wellcome Department of Imaging Neuroscience, London, UK). Each stimulus was presented at least 5 times; most stimuli were presented 8-10 times. The stimulus duration for most experiments was $1000 \mathrm{~ms}$. All stimuli were random dot patterns presented in circular apertures on a dark background of $0.024 \mathrm{~cd} / \mathrm{m}^{2}$. The dot density was 0.5 dots per degree ${ }^{2}$. Each dot was $0.1^{\circ}$ wide, with a luminance between 0.28 and $61.9 \mathrm{~cd} / \mathrm{m}^{2}$ depending on the stimulus contrast. For the coherence stimuli and the surround tuning stimuli, the dot luminance was kept at the highest value, $61.9 \mathrm{~cd} / \mathrm{m}^{2}$.

Because most standard definitions of contrast apply only to periodic stimuli, we used the SD of the luminance or root mean square (RMS) contrast as our measure of stimulus contrast (Martínez-Trujillo and Treue, 2002; Pack et al., 2005). In all our experiments, the term "contrast" indicates the RMS definition of contrast:

$$
C_{\mathrm{RMS}}=\sqrt{\sum_{i}\left(L_{i}-L_{\mu}\right)^{2} / n}
$$

where $L_{i}$ is the luminance of the individual pixels, $L_{\mu}$ is the mean luminance of the display, and $n$ is the number of pixels. For random dot patterns, the RMS contrast metric describes the relationship between the stimulus and its effectiveness at contrast adaptation better than many other contrast metrics including Michelson contrast (Moulden et al., 1990).

Coherence data were recorded with variable coherence dots stimuli in which the coherence was controlled by randomly replotting noise dots on each frame (Morgan and Ward, 1980; Newsome et al., 1989). For example, in a $12.8 \%$ coherence stimulus, on each frame $12.8 \%$ of the dots were randomly selected to be replotted with the same direction and displacement and the other $87.2 \%$ were randomly assigned new positions. This random replotting meant that the dot lifetimes followed an exponential decay that varied with the stimulus coherence.

In addition to our standard stimulus, we also recorded responses from a small number of neurons (see supplemental Fig. 1, available at www. jneurosci.org as supplemental material) to a second variable coherence dots stimulus in which all dots had an infinite lifetime. On every frame, all of the dots moved in a random walk at the stimulus speed; coherence was controlled by varying the proportion of dots that moved in the coherent direction instead of random directions. All other properties of the stimulus were identical with the standard stimulus.

\section{Data analysis}

All data analysis except for spike sorting was performed using MATLAB (MathWorks). Mean spike rates and SEs for size and direction tuning curves were calculated over time intervals starting $40 \mathrm{~ms}$ after stimulus onset and ending at stimulus offset.
Suppression index. A suppression index, SI, measuring the percentage surround suppression for each size tuning curve was calculated using the following equation:

$$
\mathrm{SI}=100 * \frac{\left(R_{\max }-R_{\text {largest }}\right)}{R_{\max }},
$$

where $R_{\max }$ is the maximum response of the neuron and $R_{\text {largest }}$ is the response of the neuron at the largest stimulus diameter, $30^{\circ}$.

Bootstrap tests. Suppression indexes measured from the same neuron under different stimulus conditions were considered significantly different if they met the following criteria: The high and low stimulus curves were resampled with replacement 10,000 times. After each resampling, new mean curves and suppression index values were calculated, generating bootstrapped distributions of suppression index values for both stimulus conditions. The bootstrapped high condition suppression index distribution was compared with the actual low condition suppression index value and the bootstrapped low condition suppression index distribution was compared with the actual high condition suppression index value. A neuron was considered to have significantly changed its suppression index only if both the high and low condition values were in the 2.5 percentile tails of the respective bootstrapped distributions.

Surround direction tuning. To calculate the population mean for the center and surround direction tuning curves, each curve was normalized to the maximum response of the neuron and rotated so that the preferred direction of the center was $0^{\circ}$. Both curves were rotated by the same amount so that the relative relationship of their direction tuning curves was maintained. The normalized and rotated tuning curves of the recorded population were then averaged.

Neurons with significant direction tuning were determined by randomly permuting the firing rates from individual stimulus presentations across all directions and recalculating a new mean firing rate and mean vector length for each permuted tuning curve. The distribution of mean vector lengths from the permuted data $(n=2000)$ was compared with the original mean vector length; a tuning curve with a mean vector length in the highest $1 \%$ of permuted data was considered to be significantly tuned.

To determine the fraction of suppressive surround input contributed by nonpreferred motion directions (see Fig. $6 \mathrm{C}$ ), we summed the response differences caused by adding the surround annulus to the center stimulus for all nonpreferred surround directions divided by the response differences across all surround directions using the following equation:

$$
\mathrm{SS}_{\mathrm{np}}=\frac{\sum_{i=2}^{n}\left(C-S_{i}\right)}{\sum_{i=1}^{n}\left(C-S_{i}\right)},
$$

where $C$ is the response to the optimal center stimulus alone and $S_{i}$ is the response to the optimal center stimulus combined with a surround annulus moving in one of $n$ possible different directions. $S_{1}$ is the response when the surround moves in the preferred direction of the center and is therefore excluded from the numerator.

\section{Results}

We recorded the activity of $97 \mathrm{MT}$ neurons from three awake, fixating macaques. To measure surround suppression, size tuning curves were collected for 53 neurons at multiple contrasts and coherences (Fig. 1 $A, B$ ). Surround direction tuning curves were also collected for most of these neurons (38 of 53) plus an additional 44 neurons. The size tuning stimuli were random dot patterns moving in the preferred direction and speed of the neuron. Surround direction tuning stimuli were composed of a central random dot pattern that moved in the preferred direction of the neuron and a surrounding annular dot pattern that varied in 
A

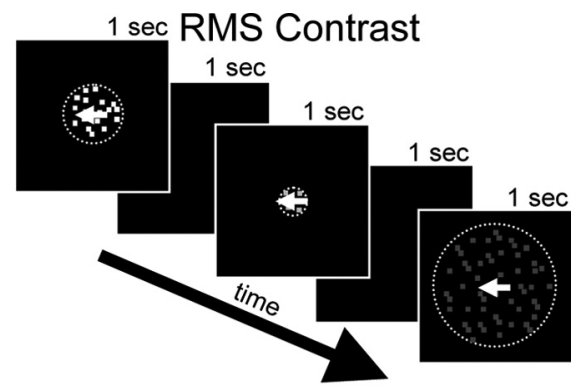

B

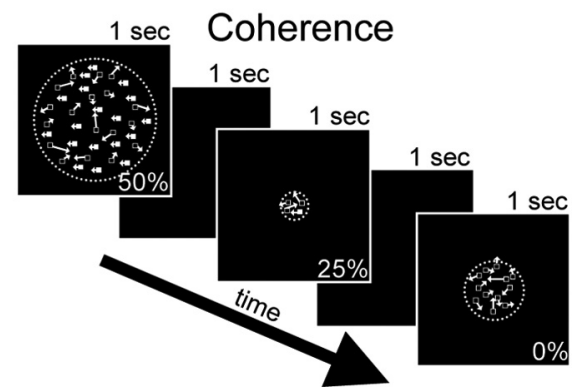

Figure 1. Visual stimuli used to measure size tuning curves. $A$, Presentation of a series of randomly interleaved dots stimuli of varying stimulus RMS contrast and size. The dots always moved in the preferred direction and speed of the neuron. Contrast stimuli were fully coherent. $\boldsymbol{B}$, Presentation of a series of randomly interleaved dots stimuli of varying motion coherence and size. The coherent dots moved in the preferred direction and speed of the neuron while the remaining dots were replotted randomly. Coherence stimuli were presented at maximum contrast. All stimulus sizes and coherences were randomly interleaved.

direction. The central patch matched the size and preferred speed of the classical receptive field estimated during hand mapping with high contrast stimuli. The surrounding annulus moved at the same speed and extended to make a total stimulus diameter of $30^{\circ}$.

\section{Surround suppression varying contrast and coherence}

Many of the MT neurons we recorded reduced the strength of their suppressive surrounds when stimulus contrast was decreased (Pack et al., 2005). Size tuning curves at multiple stimulus contrasts are shown for two example neurons in Figure 2, $A$ and $B$. As contrast decreased, the size tuning curves changed from strong surround suppression to weaker surround suppression or, in some cases, facilitation. The change in the shape of the curve at low contrasts is mainly attributable to decreased neuronal responsiveness for the smaller sizes; however, the shape change can also arise from increased responses to large low contrast stimuli. As illustrated in the example neurons, the reduction of surround suppression at low contrasts can produce the counterintuitive result that, as stimulus contrast decreases, surround-suppressed neurons increase their firing rates for large stimuli.

To quantify the amount of surround suppression present at each contrast, we calculated a suppression index for each size tuning curve. The suppression index was defined as the percentage decrease in neuronal response between the preferred stimulus size (i.e., the size that elicits the greatest response) and the largest stimulus, $30^{\circ}$ in diameter. A neuron with a suppression index of $100 \%$ would be completely suppressed by the largest stimulus, whereas a neuron with a suppression index of $0 \%$ would have no observed surround suppression. To examine how changing contrast affects surround suppression, we plotted the suppression index as a function of stimulus contrast. The suppression index of
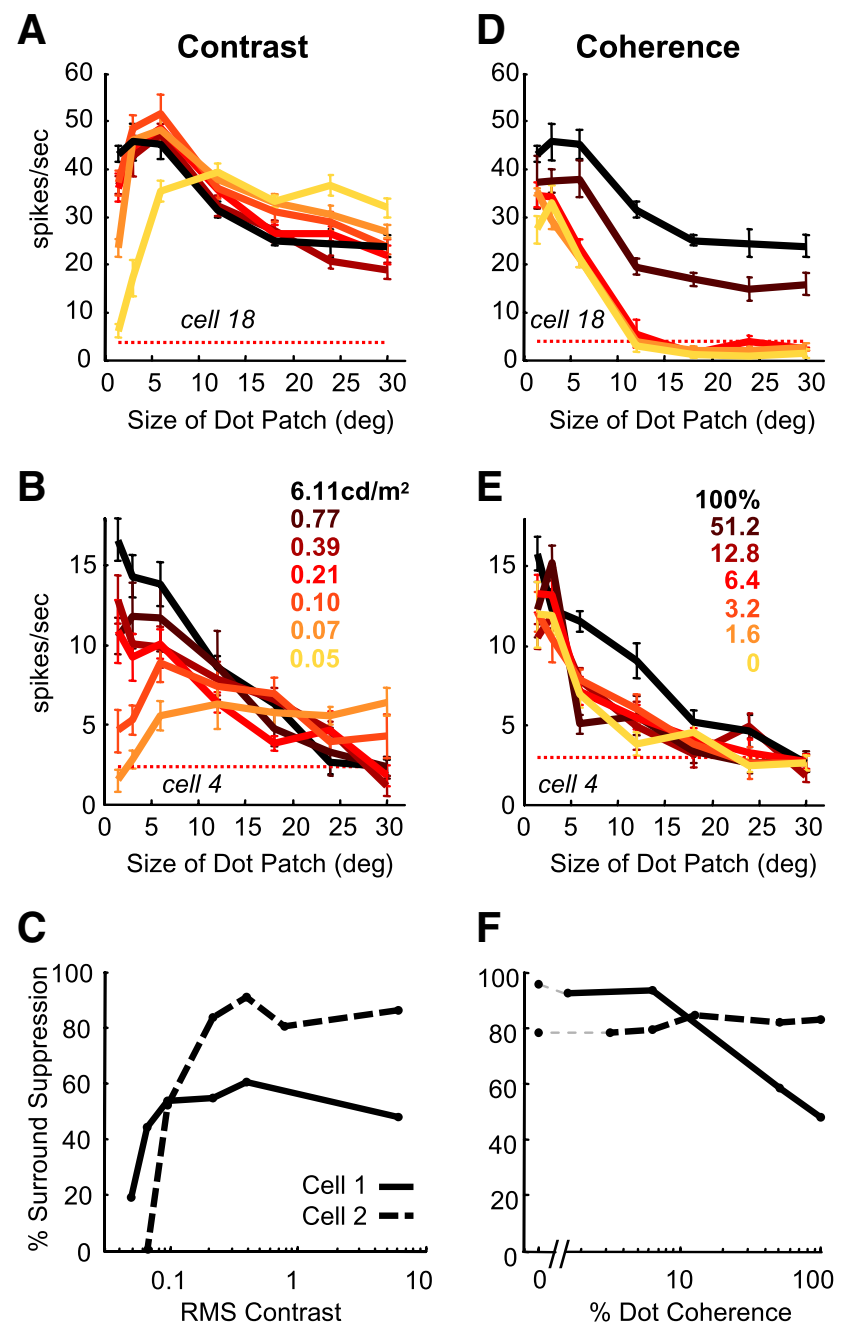

Figure 2. The effect of contrast and coherence on MT size tuning. $\boldsymbol{A}, \boldsymbol{B}$, Size tuning curves for two MT neurons measured at multiple contrasts. Curves are color-coded from high (black) to low (yellow); not all contrasts or coherences were recorded for each cell. $C$, Surround suppression index as a function of stimulus contrast for the two example cells. $\boldsymbol{D}$, $\boldsymbol{E}$, Size tuning curves at multiple coherences for the same two neurons in $\boldsymbol{A}$ and $\boldsymbol{B}$, respectively. $\boldsymbol{F}$, Surround suppression index as a function of stimulus coherence for the two example cells. All error bars represent SEM.

the upper example cell decreased from 48 to $19 \%$ as RMS contrast (see Materials and Methods) decreased from 3.09 to $0.01 \mathrm{~cd} / \mathrm{m}^{2}$. The lower example cell had a high contrast suppression index of $88 \%$. For this cell, the suppression index decreased to $0 \%$ at the lowest contrast, indicating a complete loss of observable suppression (Fig. 2C). These changes in surround suppression were significant for both cells ( $p<0.05$, bootstrap test) (see Materials and Methods).

Our manipulations of size and contrast confirmed that MT neurons decreased the relative strength of their surround suppression at low contrasts. However, decreasing contrast is only one of many ways to decrease stimulus strength. If MT neurons decrease their surround suppression as part of a general strategy for processing weak stimuli, then we would expect that other manipulations of stimulus strength should also reduce surround suppression. To test this, we remeasured size tuning curves in the same MT neurons and varied stimulus strength by manipulating motion coherence instead of contrast (Fig. $2 D, E$ ). Contrast was kept at the maximum value $\left(6.11 \mathrm{~cd} / \mathrm{m}^{2}\right)$. Lowering motion coherence reduced responses to stimuli confined to the receptive 
A

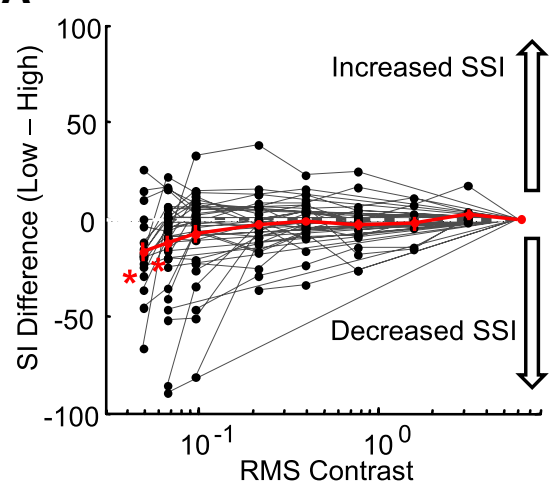

B
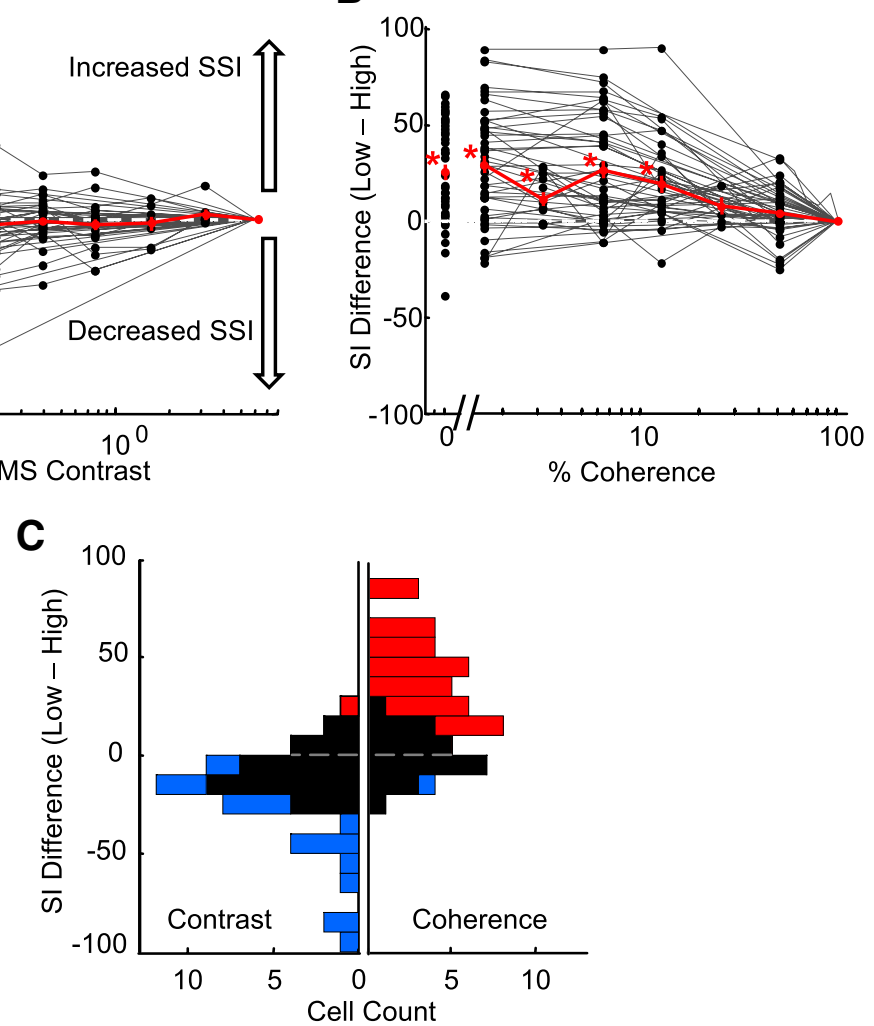

Figure 3. Differences in surround suppression across stimulus conditions for contrast and coherence. $\boldsymbol{A}$, Change in suppression index for contrast size tuning curves relative to the high contrast curve. Since the high condition suppression index is not shifted relative to itself, the curves of all neurons end at zero, by definition. Each gray line represents a separate neuron; the red line is the population mean. The asterisks indicate values significantly different from zero ( $p<0.007$, one-sided $t$ test adjusted for multiple comparisons). Error bars represent SEs. B, Change in suppression index for coherence size tuning curves relative to the high coherence curve. $\boldsymbol{C}$, Histogram of the suppression index difference between the lowest and the highest condition (coherence or contrast). The left-facing bars represent contrast measurements. The right-facing bars represent coherence measurements. Blue indicates significantly less suppression in the low condition. Red indicates significantly more suppression in the low condition. Black indicates no significance difference.

the average decreases in suppression index were -18 and $-16 \%$, respectively. For the same population of MT cells, lowering coherence caused a positive shift in the mean suppression index of the population, showing increased surround suppression (Fig. 3B). The mean change in suppression index for coherence was significantly greater than zero for the four lowest coherences: $0,1.6,3.2$, and $6.4 \%$, with shifts of $25,28,11$, and $26 \%$, respectively ( $p<0.0083$, one-sided $t$ test adjusted for multiple comparisons).

The distribution of differences in suppression index between the highest and lowest contrast conditions measured for each cell is shown in the left histogram of Figure $3 C$. The right histogram shows the similar plot for coherence. We used a bootstrap method to test for significant differences between high and low suppression index values (see Materials and Methods). Thirty-nine percent (18 of 46) of neurons had significantly less surround suppression at low contrast and only one cell $(2 \%)$ had significantly more surround suppression at low contrast. When we varied coherence, $55 \%$ (29 of 53 ) of the neurons had significantly greater surround suppression at low coherence and only one cell $(2 \%)$ had significantly less surround suppression. Our results show that, across our population of MT neurons, lowering contrast tends to decrease surround suppression and lowering motion coherence tends to increase surround suppression. Thus, the reduction by the visual system of surround suppression to

field center (Britten and Newsome, 1998), but the decline was even greater for large stimuli, indicating increased surround suppression.

Comparing the size tuning curves recorded from each neuron, it is apparent that stimuli of low coherence affect surround suppression in MT in a qualitatively different way than the decreased suppression observed with low contrast stimuli. Figure $2 \mathrm{~F}$ shows how neuronal surround suppression changed as a function of stimulus coherence. Although low contrasts produced less suppression, surround suppression was maintained or increased as stimulus coherence was lowered. Surround suppression did not decrease even when stimulus coherence was reduced to $0 \%$, so that the stimulus, on average, contains equal motion energy in all directions.

To examine the effects of contrast and coherence on surround suppression across the population, we calculated the change in suppression index measured for each curve relative to the suppression index measured for that neuron at the highest contrast or coherence. A positive shift indicates an increase in surround suppression relative to the highest condition, whereas a negative shift indicates a decrease in suppression. The mean suppression index shift of the population (red line) for contrast shows a negative shift (Fig. $3 A$ ), with the mean shift significantly less than zero for the two lowest contrasts ( $p<0.0071$, one-sided $t$ test adjusted for multiple comparisons). For these contrasts, 0.01 and $0.02 \mathrm{~cd} / \mathrm{m}^{2}$, improve integration is not a general strategy implemented whenever an important stimulus feature is weakened. Instead, it appears that the reduction of surround suppression is restricted to certain conditions, one of which is low contrast. Although the suppression index is a useful metric for quantifying the strength of surround suppression, it only captures information about the ratio of two data points on the size tuning curve: the peak response and the response to the largest stimulus size. Consequently, our use of suppression indexes to make comparisons across stimulus conditions obscures potential differences in absolute firing rates. Because we have found that some MT neurons fire more spikes for large stimuli as contrast decreases, we wanted to compare the firing rates between the high and low coherence size tuning curves. For each neuron, we calculated response difference curves, for coherence and for contrast, by subtracting the responses of the low condition curve from the responses of the high condition curve, and then normalizing the resulting curve to the maximum firing rate of the neuron. The contrast difference curve for the example neuron in Figure 2, $B$ and $E$, shows that the response of the neuron was largest for small stimuli at high contrast, but reversed to a preference for large stimuli at low contrast (Fig. $4 A$, top panel). In comparison, the coherence difference curve for the same cell shows relatively similar firing rates at high and low coherences for all stimulus sizes (Fig. $4 B$, top panel). We color-coded the response difference curves for each cell and plot- 

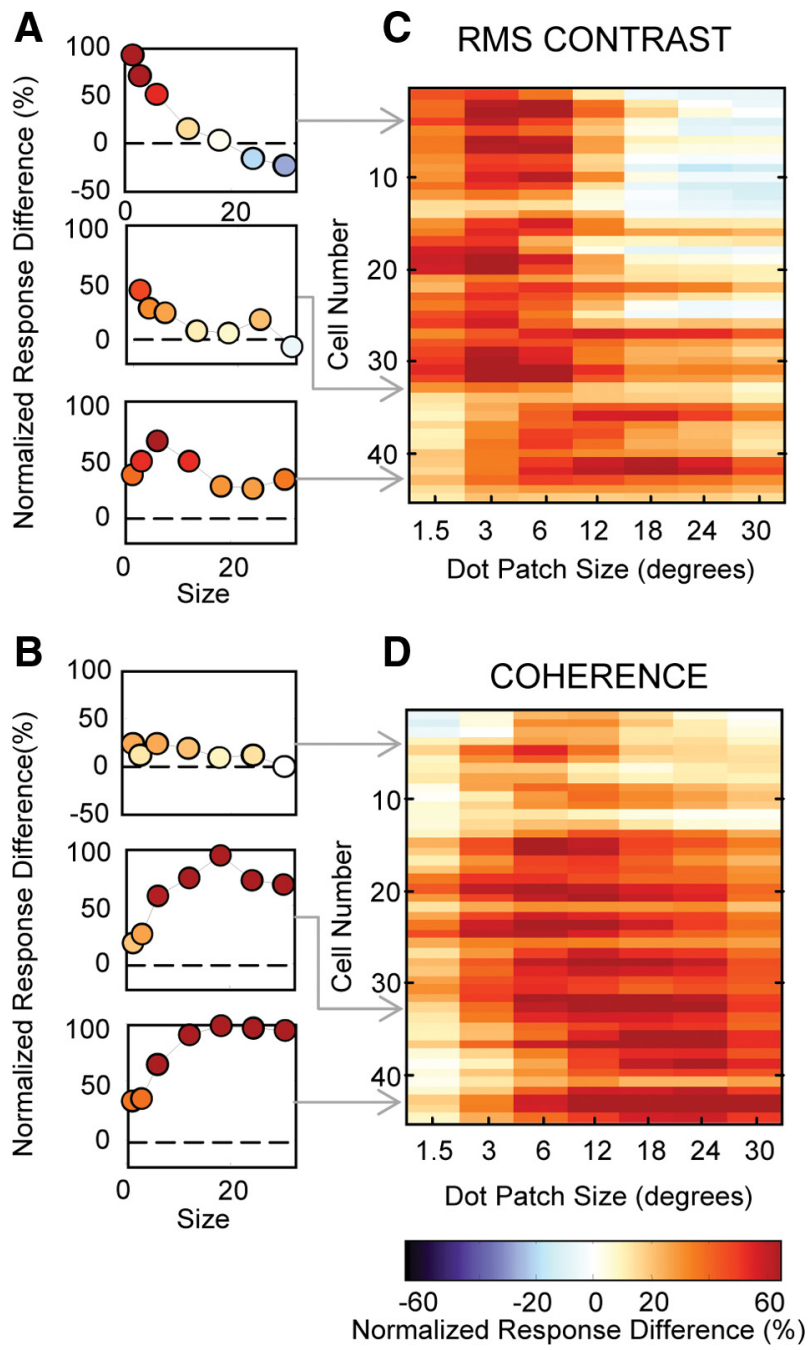

Figure 4. Differences in firing rates between high and low conditions for contrast and coherence in MT. $\boldsymbol{A}$, Difference curves resulting from subtracting low from high contrast curves, normalized to the maximum firing rate. Positive values indicate larger responses at high contrast, and negative values indicate larger responses at low contrast. $\boldsymbol{B}$, Analogous difference curves for coherence size tuning. $\boldsymbol{C}$, Contrast difference tuning curves for every neuron in the population, ordered by high coherence suppression index. The blue regions represent increased responses in the low condition, white represents similar responses, and orange represents decreased responses. Population plots were smoothed with a Gaussian (1 pixel SD) to highlight trends in the population data. $\boldsymbol{D}$, Coherence difference curves for every neuron in the population, ordered by high coherence suppression index.

ted the entire population ordered by their high coherence suppression index values (Fig. $4 C$, contrast; $D$, coherence). The cells with the greatest surround suppression at high contrast had greater firing rates for large low contrast stimuli (Fig. $4 C$, bluewhite region on the right), but the same cells generally decreased their firing rates for low coherence stimuli of the same size (redorange coloring in the same section of Fig. $4 D$ ). The weaker color saturation at the top of Figure $4 D$ shows that cells with the strongest surround suppression were the cells that tended to have the smallest differences between their high and low coherence curves. As for the example cell in Figure 2D, most cells were suppressed by low coherence but the difference in firing rate was greater for large sizes. Firing rates were relatively similar for high and low coherence stimuli when stimuli were small (Fig. $4 D$, light coloring on the left), but low coherence stimuli produced greater reductions in response rate as size increased, consistent with greater surround suppression (Fig. 4D, darker coloring).
One potential explanation for the increase in surround suppression at low coherences is that the flicker produced by the limited lifetime of the dots (see Materials and Methods) activates more surround suppression. The low coherence limited lifetime stimuli also contain a broad range of dot speeds that could activate suppression to nonpreferred speeds in the surround (Xiao et al., 1998). Our results do not depend on either feature of limited lifetime noise dots, as we obtained similar results using infinitelifetime dots with nonflickering, single-speed "random walk" noise (Nawrot and Sekuler, 1990) in a subset of our neurons (supplemental Fig. 1, available at www.jneurosci.org as supplemental material).

\section{Surround direction tuning}

Another possible explanation for the increase in surround suppression observed at low coherences is that these stimuli activate surround suppression by nonpreferred directions of motion. The response of a neuron depends on the relative activation of its center and surround. A surround with broader direction tuning than the center would "see" more of the motion energy in a low coherence stimulus and therefore be relatively more activated than the center. This does not necessarily mean that low coherence stimuli are optimal for the surround, only that they are relatively less poor for the surround than for the center. We examined the direction tuning of surround suppression in MT by measuring responses to an annular surround stimulus that varied in direction while a preferred direction stimulus moved within the classical receptive field (Fig. 5A). The stimuli were of high contrast and 100\% coherent. We found that many MT neurons were strongly suppressed by the surround patch regardless of its direction of motion. Figure 5, $B$ and $C$, shows polar plots of the direction tuning of the center alone (in blue) and the surround (in red) for two example cells. Points on the surround direction tuning plots show the mean response of the neuron when the surround dots moved in that direction while the center was stimulated with dots moving in the preferred direction. Thus, these surround responses should be compared with the center response to the preferred direction, which is indicated by a blue, dashed circle in Figure 5, $B$ and $C$. Since surround suppression reduces neuronal activity, a smaller response indicates greater suppression. Some neurons had no observable suppression when the surround dots moved in the preferred direction but were strongly suppressed when the surround dots moved in other directions (Fig. 5C).

The mean center and surround direction tuning curves for the population show that, on average, neurons were suppressed strongly in all directions, with the mean suppression varying between 51 and $61 \%$ depending on the direction (Fig. $6 A)$. However, the population mean is not a useful metric if the population of MT surrounds is too heterogeneous, with some cells strongly suppressed and others strongly facilitated by surround motion or if surrounds were all strongly tuned but varied in their preferred direction. Although some surrounds were indeed strongly tuned, we found that in general this was not the case. We examined the breadth of direction tuning by calculating the mean vector length for both tuning curves (Fig. $6 \mathrm{~B}$ ). A circular tuning curve has a mean vector length of 0 , whereas a neuron that responded to only one direction of motion would have a mean vector length of 1 . The median mean-vector lengths across the population were 0.58 for the center and 0.20 for the surround. All of the tuning curves for the receptive field centers were significantly tuned 
A

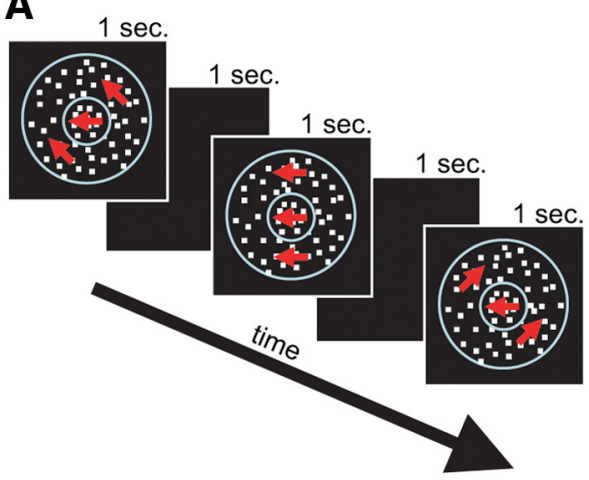

B

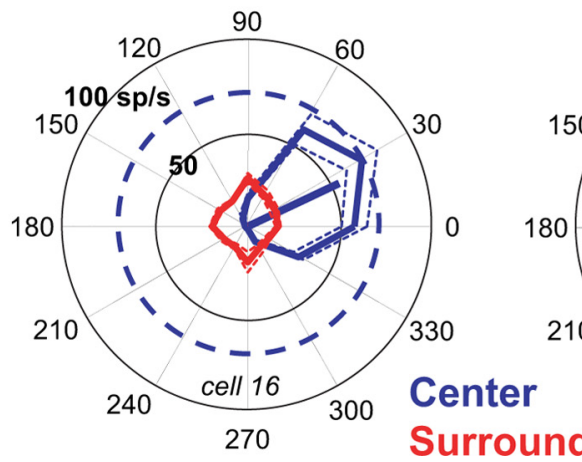

C

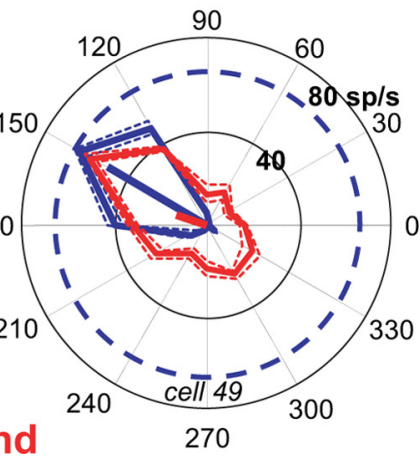

Figure 5. Surround direction tuning: example cells. $\boldsymbol{A}$, Stimuli used to measure surround direction tuning. The center receptive field contained a dot patch moving in the preferred direction and speed of the neuron. The surround receptive field was stimulated with an annular dot patch (outer diameter, $30^{\circ}$ ) that varied in direction. Both patches were high contrast and high coherence. $\boldsymbol{B}$, C, Direction tuning of the center (blue) and surround (red) for two MT neurons. For the surround direction tuning curves (red), the center was always stimulated with dots moving in the preferred direction of the neuron. Thus, the responses when the surround moved in different directions should be compared with the blued dashed circle indicating the response to the center only at the preferred direction. The dotted lines indicate SEs.

A

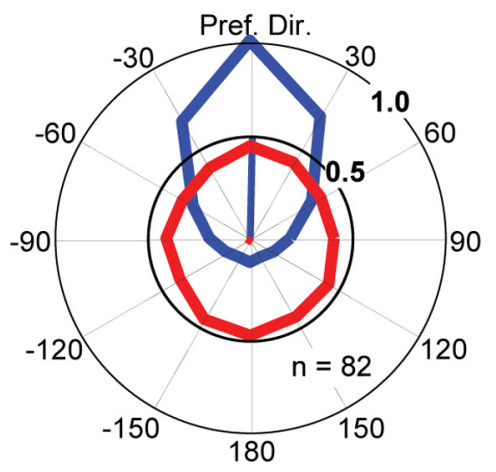

B

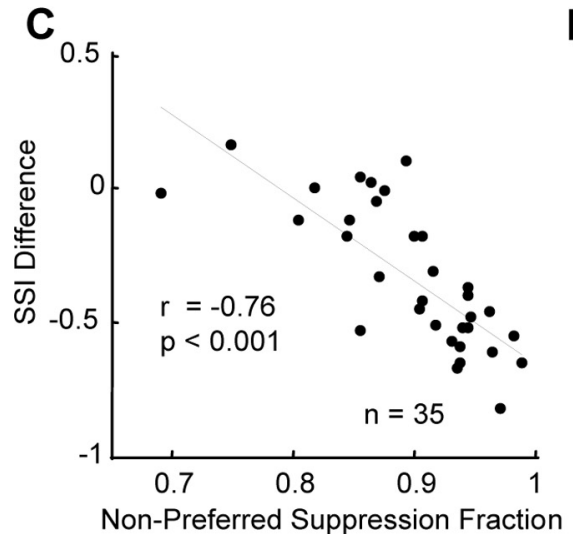

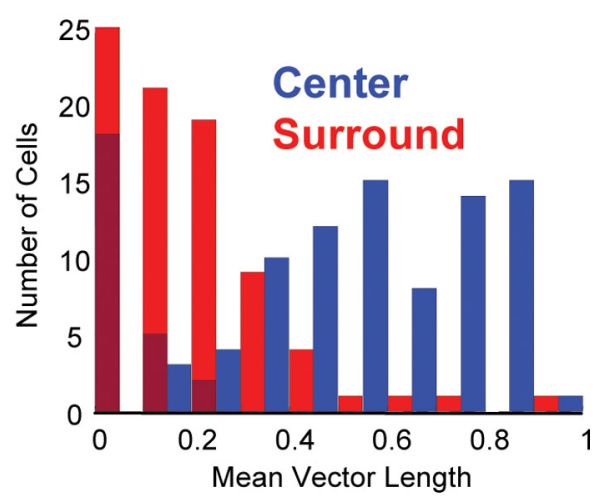

D

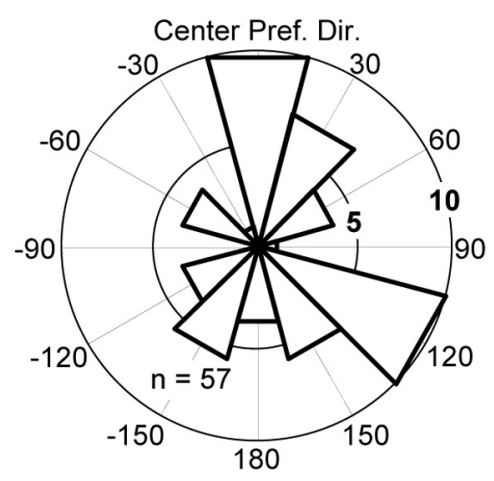

Figure 6. Surround direction tuning: population data. $\boldsymbol{A}$, Population mean center (blue) and surround (red) direction tuning curves. Before averaging, the tuning curves of each neuron were normalized and rotated so that the preferred direction of the center was $90^{\circ}$ (up) and the magnitude of the response to this direction was 1. $\boldsymbol{B}$, Histogram of mean vector lengths for center (blue) and surround (red) direction tuning curves. The darker red bars indicate neurons whose surrounds were not significantly direction selective. $C$, Fraction of surround suppression from nonpreferred directions versus the difference in suppression index between the high and low coherence conditions. The black line is the regression line $(r=-0.76 ; p<0.001)$. $\boldsymbol{D}$, Histogram of preferred surround directions for neurons with significantly tuned surrounds. This distribution did not differ significantly from circular uniformity (Rayleigh's test, $p=0.23$ ).

(i.e., had mean vector lengths significantly greater than zero; $p<0.01$, permutation test), but $30 \%$ (25 of 82 ) of the surround direction tuning curves were not $(p>0.05)$.

Since low coherence stimuli contain motion energy in all directions, they should more effectively activate the more broadly tuned surround mechanism. For example, a neuron with a selective surround might be strongly facilitated by surround motion in the null direction, but strongly suppressed by other directions of surround motion. A low coherence stimulus would activate both the suppression and the facilitation; the effect on the low coherence size tuning of a neuron would depend on which exerted more influence. Insofar as the neuron was suppressed by more, different surround directions, low coherence stimuli should become more effective at activating it. A simple expectation of the response of the surround to low coherence dots could be obtained by using the surround direction tuning curve to find the mean surround modulation across all directions. Fifty-six percent (47 of 83 ) of the neurons had $>50 \%$ suppression on average across all surround directions. Only 7\% (6 of 83) of the neurons had surrounds that were on average more facilitatory than suppressive. The strong suppression of MT cells by nonpreferred directions of surround motion explains why most MT cells increase their surround suppression at low coherences. Consistent with this, we found that the fraction of the surround suppression of a neuron arising from nonpreferred directions of motions (see Materials and Methods) was significantly correlated with larger increases in surround suppression at low coherence $(r=-0.76 ; p<0.001)$ (Fig. 6C). Thus, the increased surround suppression observed at low coherences appears to be attributable to suppressive influences that pool broadly across motion direction in the surround. Across the population of cells with significantly tuned surrounds ( $n=57)$, we found no systematic bias for preferred directions of surround motion relative to the preferred direction of the center (Fig. 6D) (Rayleigh's test, $p=0.23$ ). 


\section{Discussion}

Our results demonstrate that the visual system does not always reduce surround suppression to increase spatial integration when an important stimulus feature is noisy. When we tested MT neurons with stochastic motion stimuli that allowed us to systematically vary the strength of the preferred motion signal while keeping the overall contrast constant, we found that low motion coherence tended to increase, not decrease, surround suppression, thus producing a less integrative surround. This somewhat surprising result is most likely attributable to the activation of surround suppression by motion in nonpreferred directions. Consistent with this, we found that the direction tuning of surround suppression in MT was, in general, extremely broad.

Previous work has shown that MT neurons are less surround suppressed for low contrast stimuli (Pack et al., 2005) and that, in general, surround effects become more integrative when weak or ambiguous motion is present in the receptive field center (Huang et al., 2007, 2008). These results have been unified by the idea that motion uncertainty leads to greater integration by MT neurons (Huang et al., 2007). Such uncertainty can be created by reducing the signal-to-noise ratio, either by lowering contrast or by adding motion noise, as was done in our experiments, or by generating motion whose direction is ambiguous because of the "aperture problem" (Marr and Ullman, 1981). In the latter case, the motion signal may be strong (e.g., produced by a high contrast, onedimensional contour viewed through a circular aperture), but its true two-dimensional direction of motion is uncertain because it is consistent with a range of possible two-dimensional directions. Technically speaking, then, one should not equate motion ambiguity with low signal-to-noise. Nevertheless, they both produce motion uncertainty, and, interestingly, the long contours that create the aperture problem tend to produce considerably lower response rates in MT neurons than do stimuli that place "terminators," such as dots and short bars, in the receptive field center (Huang et al., 2008). In this sense, all of the classes of stimuli that produce motion uncertainty (low contrast, low coherence, and long contours) tend to be less effective at driving MT neurons than those that do not.

As all three classes of stimuli producing motion uncertainty have been shown to increase motion integration perceptually (Lorenceau and Shiffrar, 1992; Watamaniuk and Sekuler, 1992; Murakami and Shimojo, 1993), we predicted that low coherence random dots would cause MT neurons to show greater facilitation for preferred direction motion in the surround or, at least, less suppression. Our results, however, ran counter to this prediction. One possible, trivial explanation lies in the different ways in which surround modulation can be studied: as facilitation versus suppression, which refers to the effect of a given surround on the firing rate of a neuron, or as integration versus antagonism, which measures the relative direction tuning of the surround with respect to the center. Although related, the two different ways of characterizing center-surround interactions are not identical-for example, antagonism is often characterized by suppression for preferred direction motion and facilitation for null direction motion (Allman et al., 1985a; Born and Tootell, 1992; Born, 2000). The experiments in which we systematically varied motion coherence did not vary the surround direction, so we cannot speak to changes in the tuning of surround modulation and, therefore, according to the above definition, cannot assess changes in integration versus antagonism that were measured by Huang et al. (2007, 2008). Nevertheless, the strong prediction of the motion uncertainty hypothesis is that preferred direction responses in the surround should become facilitatory or, at least, less suppressive as the stimulus signal-to-noise ratio decreases, and we found the exact opposite. We interpret this to mean that the center-surround interaction is not quite as sophisticated as one might have hoped, and that this shortcoming points to a more mechanistic picture of the surround.

\section{Surrounds as a manifestation of normalization}

The general picture of the MT surround that emerges from this study and others is that it appears to pool signals with much less selectivity than does the center along several different dimensions, in line with the notion that it is but one manifestation of divisive normalization (Heeger, 1992; Carandini et al., 1997; Cavanaugh et al., 2002). In fact, the low selectivity of a "normalization pool" along three dimensions-retinotopy, direction, and input stream - can potentially explain many features of receptive field surrounds. The broader retinotopic pooling is what produces the phenomenon of a larger, nonclassical spatial surround in the first place. Less selective pooling of directions accounts for stronger suppression at low coherences since these stimuli, which have motion energy distributed across all directions, would be relatively more effective for the less selective surround than for the more selective center. Finally, the reduced surround suppression at low contrast might simply reflect a lower contrast sensitivity of the surround, relative to that of the center. This could be explained by the inclusion of inputs from both the $\mathrm{M}$ (magnocellular) and $\mathrm{P}$ (parvocellular) streams in the surround, although other explanations are possible given the nonlinear contrast responses of the neurons that provide input to MT (J. M. Tsui and C. C. Pack, unpublished observations). If the center mechanism selectively integrates $\mathrm{M}$-stream input, one would expect high contrast sensitivity and relatively rapid saturation of responses, as has been documented previously (Sclar et al., 1990). Inclusion of P-stream input in the normalization pool comprising the surround could provide additional drive beyond the point where $\mathrm{M}$-stream responses saturate, thus maximizing the influence of the surround at higher contrasts. Although this idea is speculative, it is supported by the M-stream domination of the so-called "direct" pathway to MT from layer 4B of V1 (Yabuta et al., 2001; Nassi and Callaway, 2006) and the mixed M and P inputs arriving in MT from either the "indirect" pathways via areas V2 and V3 (Yabuta et al., 2001) or via layer 6 Meynert cells of V1 (Nassi et al., 2006). It is further supported by a modest, but significant, decrease in surround suppression seen when the indirect inputs to MT are reversibly inactivated (C. R. Ponce, J. N. Hunter, C. C. Pack, S. G. Lomber, and R. T. Born, unpublished observations).

\section{Suggested modifications to existing models}

Several groups have proposed recurrent network models of center-surround interactions (Somers et al., 1998; Schwabe et al., 2006; Huang et al., 2008) that share two key features governing the balance of excitation and inhibition in cortical circuits. In particular, they postulate that inhibitory mechanisms have (1) a higher threshold, requiring more input drive before they respond, and (2) a steeper gain function such that, once activated, they rapidly outstrip excitation and suppress neural responses. This has the effect that surrounds can appear to be either suppressive or facilitatory depending on the overall strength of input to the circuit, and can account for important phenomena, such as the contrast dependence of surround suppression (Pack et al., 2005; this study) and the differential effects of surround stimuli when center motion is ambiguous (Huang et al., 2007, 2008). In 
their current forms, however, these models are unable to account for the results we obtained with variable coherence motion stimuli in which, for many MT neurons (Fig. 2 B), a low coherence stimulus provides weaker overall drive (as evidenced by the lower firing rate to the stimulus restricted to the receptive field center) yet nevertheless evokes stronger suppression as it invades the surround. We propose that this can be easily remedied if the broader tuning of the surround mechanism is taken into account. From this perspective, the basic relationships between excitation and inhibition in the recurrent network models need not change; what would change is that the inhibitory mechanism (referred to as the "normalization pool" above) would sum inputs activated by a larger range of visual stimuli. This is consistent both with our measurements of the broad direction tuning of MT surrounds and with the finding that, at least in V1, inhibitory interneurons are very poorly tuned for orientation (Sohya et al., 2007; Niell and Stryker, 2008).

One potential problem with the above argument is that it is not clear whether low coherence stimuli in fact provide weaker input to the center mechanism of MT neurons. It is widely believed that the lower firing rates to such stimuli are attributable to "motion opponency" - the property by which responses to preferred direction stimuli confined to the receptive field center are suppressed when null direction motion is added-and that this is computed within MT. Evidence that motion opponency is an MT computation rests primarily on the observation that the property is common in MT but rare in V1 (Snowden et al., 1991; Qian and Andersen, 1994). However, there is strong motion opponency in a significant minority of V1 cells [for example, see Snowden et al. (1991), their Fig. 4; Qian and Andersen (1994), their Fig. 15], and it is possible that these motion-opponent neurons are the ones that project to MT. There is certainly precedent for extremely high selectivity in which V1 neurons project to MT in terms of their direction selectivity [Movshon and Newsome (1996), their Fig. 4]. A second difficulty in ascribing effects to motion opponency within the center is that it is impossible to activate only the center, since it is clear that the surround is spatially continuous across the receptive field (DeAngelis et al., 1994; Sceniak et al., 2001; Cavanaugh et al., 2002). That is, the true shape of the surround is a Gaussian not an annulus. This means that a broadly direction tuned suppressive "surround" mechanism would also contribute to motion opponency observed in the center. From this perspective, both the suppressive surround and motion opponency would be different manifestations of a single normalization mechanism.

\section{References}

Allman J, Miezin F, McGuinness E (1985a) Direction- and velocity-specific responses from beyond the classical receptive field in the middle temporal visual area (MT). Perception 14:105-126.

Allman J, Miezin F, McGuinness E (1985b) Stimulus specific responses from beyond the classical receptive field: neurophysiological mechanisms for local-global comparisons in visual neurons. Annu Rev Neurosci 8:407-430

Barlow HB (1959) Sensory mechanisms, the reduction of redundancy, and intelligence. In: The Mechanisation of thought processes (Her Majesty's Stationery Office, ed), pp 537-559. London: Her Majesty's Stationery Office.

Barlow HB (1961) Possible principles underlying the transformations of sensory messages. In: Sensory communication (Rosenblith WA, ed), pp 217-234. Cambridge, MA: MIT.

Barlow HB, FitzHugh R, Kuffler SW (1957) Changes of organization of the receptive fields of the cat's retina during dark adaptation. J Physiol 137:338-354

Born RT (2000) Center-surround interactions in the middle temporal visual area of the owl monkey. J Neurophysiol 84:2658-2669.
Born RT, Tootell RB (1992) Segregation of global and local motion processing in primate middle temporal visual area. Nature 357:497-499.

Britten KH, Newsome WT (1998) Tuning bandwidths for near-threshold stimuli in area MT. J Neurophysiol 80:762-770.

Britten KH, Shadlen MN, Newsome WT, Movshon JA (1992) The analysis of visual motion: a comparison of neuronal and psychophysical performance. J Neurosci 12:4745-4765.

Buracas GT, Albright TD (1996) Contribution of area MT to perception of three-dimensional shape: a computational study. Vision Res 36:869-887.

Carandini M, Heeger DJ, Movshon JA (1997) Linearity and normalization in simple cells of the macaque primary visual cortex. J Neurosci 17:8621-8644.

Cavanaugh JR, Bair W, Movshon JA (2002) Nature and interaction of signals from the receptive field center and surround in macaque $\mathrm{V} 1$ neurons. J Neurophysiol 88:2530-2546.

DeAngelis GC, Freeman RD, Ohzawa I (1994) Length and width tuning of neurons in the cat's primary visual cortex. J Neurophysiol 71:347-374.

Heeger DJ (1992) Normalization of cell responses in cat striate cortex. Vis Neurosci 9:181-197.

Huang X, Albright TD, Stoner GR (2007) Adaptive surround modulation in cortical area MT. Neuron 53:761-770.

Huang X, Albright TD, Stoner GR (2008) Stimulus dependency and mechanisms of surround modulation in cortical area MT. J Neurosci 28:13889-13906.

Kapadia MK, Westheimer G, Gilbert CD (1999) Dynamics of spatial summation in primary visual cortex of alert monkeys. Proc Natl Acad Sci U S A 96:12073-12078.

Levitt JB, Lund JS (1997) Contrast dependence of contextual effects in primate visual cortex. Nature 387:73-76.

Lorenceau J, Shiffrar M (1992) The influence of terminators on motion integration across space. Vision Res 32:263-273.

Marr D, Ullman S (1981) Directional selectivity and its use in early visual processing. Proc R Soc Lond B Biol Sci 211:151-180.

Martínez-Trujillo J, Treue S (2002) Attentional modulation strength in cortical area MT depends on stimulus contrast. Neuron 35:365-370.

Morgan MJ, Ward R (1980) Conditions for motion flow in dynamic visual noise. Vision Res 20:431-435.

Moulden B, Kingdom F, Gatley LF (1990) The standard deviation of luminance as a metric for contrast in random-dot images. Perception 19:79-101.

Movshon JA, Newsome WT (1996) Visual response properties of striate cortical neurons projecting to area MT in macaque monkeys. J Neurosci 16:7733-7741.

Murakami I, Shimojo S (1993) Motion capture changes to induced motion at higher luminance contrasts, smaller eccentricities, and larger inducer sizes. Vision Res 33:2091-2107.

Nassi JJ, Callaway EM (2006) Multiple circuits relaying primate parallel visual pathways to the middle temporal area. J Neurosci 26:12789-12798.

Nassi JJ, Lyon DC, Callaway EM (2006) The parvocellular LGN provides a robust disynaptic input to the visual motion area MT. Neuron 50:319-327.

Nawrot M, Sekuler R (1990) Assimilation and contrast in motion perception: explorations in cooperativity. Vision Res 30:1439-1451.

Newsome WT, Britten KH, Movshon JA (1989) Neuronal correlates of a perceptual decision. Nature 341:52-54.

Niell CM, Stryker MP (2008) Highly selective receptive fields in mouse visual cortex. J Neurosci 28:7520-7536.

Pack CC, Hunter JN, Born RT (2005) Contrast dependence of suppressive influences in cortical area MT of alert macaque. J Neurophysiol 93:1809-1815.

Polat U, Mizobe K, Pettet MW, Kasamatsu T, Norcia AM (1998) Collinear stimuli regulate visual responses depending on cell's contrast threshold. Nature 391:580-584.

Qian N, Andersen RA (1994) Transparent motion perception as detection of unbalanced motion signals. II. Physiology. J Neurosci 14:7367-7380.

Sceniak MP, Ringach DL, Hawken MJ, Shapley R (1999) Contrast's effect on spatial summation by macaque V1 neurons. Nat Neurosci 2:733-739.

Sceniak MP, Hawken MJ, Shapley R (2001) Visual spatial characterization of macaque V1 neurons. J Neurophysiol 85:1873-1887. 
Schwabe L, Obermayer K, Angelucci A, Bressloff PC (2006) The role of feedback in shaping the extra-classical receptive field of cortical neurons: a recurrent network model. J Neurosci 26:9117-9129.

Sclar G, Maunsell JH, Lennie P (1990) Coding of image contrast in central visual pathways of the macaque monkey. Vision Res 30:1-10.

Snowden RJ, Treue S, Erickson RG, Andersen RA (1991) The response of area MT and V1 neurons to transparent motion. J Neurosci 11:27682785.

Sohya K, Kameyama K, Yanagawa Y, Obata K, Tsumoto T (2007) GABAergic neurons are less selective to stimulus orientation than excitatory neurons in layer II/III of visual cortex, as revealed by in vivo functional $\mathrm{Ca}^{2+}$ imaging in transgenic mice. J Neurosci 27:2145-2149.
Somers DC, Todorov EV, Siapas AG, Toth LJ, Kim DS, Sur M (1998) A local circuit approach to understanding integration of long-range inputs in primary visual cortex. Cereb Cortex 8:204-217.

van Doorn AJ, Koenderink JJ (1984) Spatiotemporal integration in the detection of coherent motion. Vision Res 24:47-53.

Watamaniuk SN, Sekuler R (1992) Temporal and spatial integration in dynamic random-dot stimuli. Vision Res 32:2341-2347.

Xiao DK, Raiguel S, Marcar V, Orban GA (1998) Influence of stimulus speed upon the antagonistic surrounds of area MT/V5 neurons. Neuroreport 9:1321-1326.

Yabuta NH, Sawatari A, Callaway EM (2001) Two functional channels from primary visual cortex to dorsal visual cortical areas. Science 292:297-300. 